\title{
The displacement state of the posterior annulus in the intervertebral disc under flexion.
}

\author{
Qing Liu ${ }^{1,2}$, Kun $\mathrm{Li}^{3}$, Zi-Jian Gao ${ }^{1,2}$, Tao Zhang ${ }^{1,2}$, Chun-Qiu Zhang ${ }^{1,2 *}$ \\ ${ }^{1}$ Tianjin Key Laboratory for Advanced Mechatronic System Design and Intelligent Control, School of Mechanical \\ Engineering, Tianjin University of Technology, Tianjin, PR China \\ ${ }^{2}$ National Demonstration Center for Experimental Mechanical and Electrical Engineering Education (Tianjin University \\ of Technology), Tianjin, PR China \\ ${ }^{3}$ School of Electronic Information Engineering, Tianjin University of Technology, Tianjin, PR China
}

\begin{abstract}
The occurrence of lumbar disc herniation is closely related to the mechanical state, and it is necessary to study the internal mechanical behavior of the intervertebral disc under different loading conditions. In this study, the unconfined experiments were conducted by applying an optimized Digital Image Correlation (DIC) technique, and the internal displacement was analysed for the intervertebral disc under axial compression with flexion position. It is found that the average axial displacement is compressive in posterior AF, and the axial displacement in the outer region is significantly lower than that in the inner region, while the axial displacement in the lower region is significantly lower than that in the upper region. It is noted that the average radial displacement is tensile in posterior $\mathrm{AF}$, and the radial displacement in the inner region is significantly lower than that in the outer region, while the radial displacement in the upper region is significantly lower than that in the lower region.
\end{abstract}

Keywords: Intervertebral disc, Annulus fibrosus, Flexion, Internal displacement, Optimized DIC technique.

\section{Introduction}

Lumbar disc herniation is the extrusion of the nucleus pulposus out of the rupture of the annulus fibrosus, and the bulging tissue squeezes the spinal nerves continuously, leading to low back pain $[1,2]$. The occurrence of lumbar disc herniation is closely related to the mechanical state, and it is necessary to study the internal mechanical behavior of the intervertebral disc under different loading conditions [3-7]. The combination of flexion and axial compression is common in daily activities, however, this kind of activities may cause the distortions to the annulus fibrosus and even the complete rupture of the posterior annulus fibrosus in acute cases [8-11]. Therefore, it is important to study the mechanics of the intervertebral disc in this damaging load condition.

The Intervertebral Disc (IVD) consists of the liquid Nucleus Pulposus (NP) surrounded by layers of the Annulus Fibrosus (AF) $[12,13]$. In the past decade, some scholars tested the stress and the strain in the intervertebral disc under load and analySed the interrelationship between the lumbar disc structure and its mechanical properties [14-18]. Recently, NT Hollingsworth et al. measured the surface strains of the posterior annulus in an intact bovine caudal segment during flexion and axial compression loading, and determined the stress state based on the measured strains [19]. Shan et al. investigated the mechanism of the disc failure in a complex posture incorporating of flexion and shear, and studied the influence of the loading rate on the disc failure [20]. O'Connell et al. noninvasively measured the internal deformations of the human discs under axial compression with flexion, neutral, and extension positions, then analysed the effect of degeneration on the lumbar intervertebral disc [21].

Our objective was to measure the displacement of the posterior annulus in the intervertebral disc under axial compression with flexion position. By applying the optimized Digital Image Correlation (DIC) technique, the displacement field was obtained by analyzing the continuous images captured by the camera. Simultaneously, the internal axial as well as radial displacement in four unique locations of the posterior annulus was quantitatively calculated: inner $\mathrm{AF}$, outer $\mathrm{AF}$, upper $\mathrm{AF}$ and lower AF.

\section{Theoretical model}

The intervertebral disc is a complex structure that transmits and distributes large loads on the spine while providing flexibility [22]. Figure 1a shows the force analysis of the intervertebral disc in the posture of forward flexion with a flexion angle of $\theta$. 


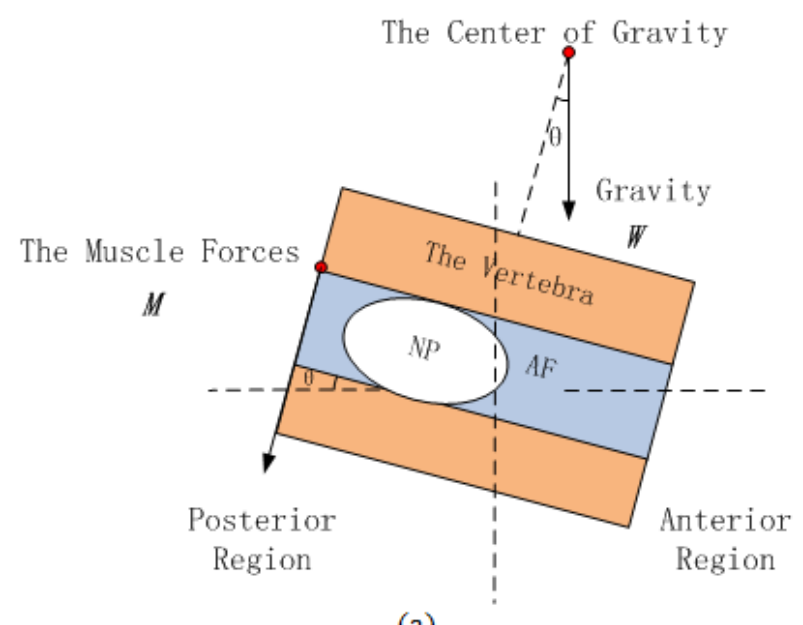

(a)

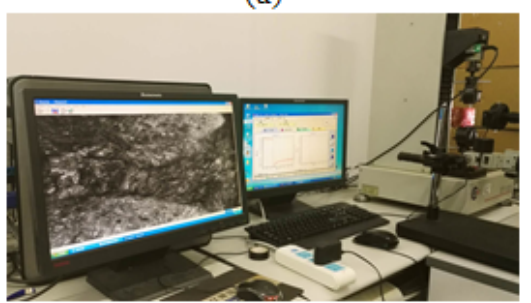

(b)

Figure 1. The force analysis and the experimental setup for the intervertebral disc in the posture of forward flexion. (a): The force analysis for the intervertebral disc; (b): The experimental setup for the intervertebral disc.

Four equilibrium functions can be obtained from Figure 1a:

$\operatorname{Sin} \theta=L_{\mathrm{w}} / L \rightarrow(1)$

$\left(w \times L_{\mathrm{w}}\right)-\left(M \times L_{\mathrm{m}}\right)=0 \rightarrow(2)$

$w \times \cos \theta+M-C \rightarrow(3)$

$w \times \sin \theta-S=0 \rightarrow(4)$

Where $\theta$ is the anteflexion angle, $C$ is the compressive force on the disc, $S$ is the shear force on the disc; $w$ is the gravity of the upper body, $L_{\mathrm{w}}$ is the arm of force for $w ; M$ is the muscle force of the straight spine muscle, $L_{\mathrm{m}}$ is the arm of force for $M$.

By solving the above four equations, the compressive force as well as the shear force on the intervertebral disc can be obtained:

$C=w \cdot\left(\cos \theta+\frac{L}{L_{m}} \cdot \sin \theta\right) \rightarrow(5)$

$S=w \times \sin \theta \rightarrow(6)$

The calculation results show that the flexion angle directly affects the force on the lumbar disc.

\section{Experiments}

Ten L3-L4 motion segments were obtained from the fresh porcine lumbar spines, and they were cut along the sagittal plane to be made into the intervertebral disc samples. The diameter as well as the height of intervertebral disc was measured to estimate the size of the intervertebral disc. A series of iron oxide nanoparticles were applied evenly to the annulus fibrosus in the sagittal plane of the samples, and they were used as the marked points to record the deformation of the intervertebral disc.

In this study, a MTF-100 loading device was applied to perform the compression test, and an optimized digital image correlation technique [23] was used to record the sequential images of the intervertebral disc samples under flexion as shown in Figure 1b. During the experiments, the samples were fixed by the fixtures with a flexion angle of $5^{\circ}$, then the samples were applied continuous compressive load with the loading rate of $1 \mathrm{~mm} / \mathrm{min}$ until the final axial strain reached $30 \%$. The displacement fields as well as the strain fields were calculated by the image processing software from the acquired images, and the load were measured by a force sensor in the loading device.

\section{Results}

The axial as well as radial displacement during static loading was performed in posterior AF to determine the magnitude and direction of AF bulging, as shown in Figure 2. The calculation results show that the average axial displacement is compressive, while the average radial strain is tensile in posterior AF.

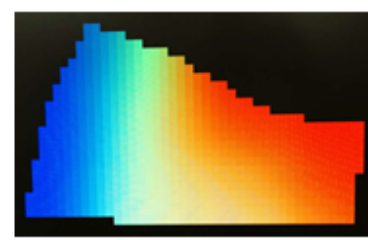

(a)

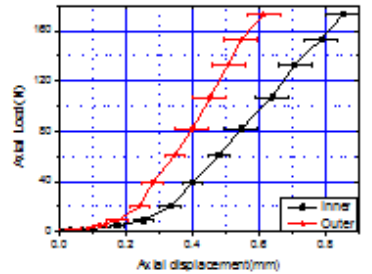

(c)

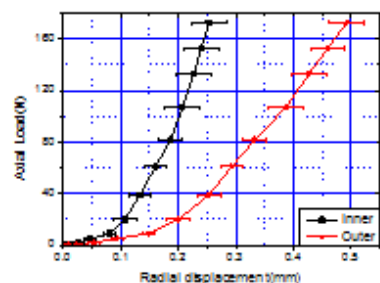

(e)

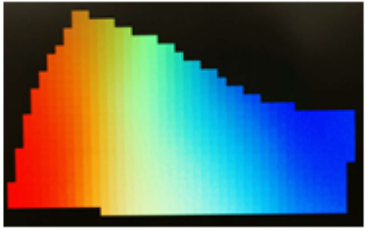

(b)

(d)

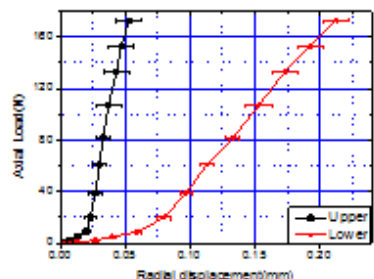

(f)

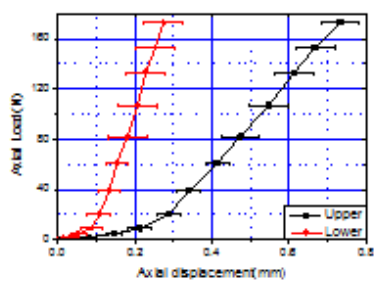

Figure 2. Displacement in posterior AF of the intervertebral disc under flexion. (a): Axial displacement field in posterior AF; (b): Radial displacement field in posterior AF; (c): Comparison of the axial displacement in inner $A F$ and outer $A F ;(d)$ : Comparison of the axial displacement in upper $A F$ and lower $A F$; (e): Comparison of the radial displacement in inner $A F$ and outer $A F$; $(f)$ : Comparison of the radial displacement in upper $A F$ and lower $A F$. 
Figures $2 \mathrm{a}$ and $2 \mathrm{~b}$ show axial and radial displacement field in posterior AF at a fix time. From Figure 2a, it can be seen that the compressive axial displacement of posterior $\mathrm{AF}$ is greater in the upper and inner regions than in the lower and outer regions. From Figure $2 b$, it can be seen that the tensile radial displacement of posterior AF is greater in the lower and outer regions than in the upper and inner regions.

Figures $2 \mathrm{c}$ and $2 \mathrm{~d}$ show axial displacement-axial load curves in different regions of the posterior AF during static loading. It is found that the displacement-load curves are nonlinear, and the displacement increases gradually with the increase of load on the posterior AF. From Figure 2c, it can be seen that the axial displacement in the outer region is significantly lower than that in the inner region. From Figure 2d, it can be seen that the axial displacement in the lower region is significantly lower than that in the upper region. The axial displacement state is in good agreement with the axial displacement field quite well, as shown in Figure $2 \mathrm{a}$.

Figures $2 \mathrm{e}$ and $2 \mathrm{f}$ show radial displacement-axial load curves in different regions of the posterior AF during static loading. It is found that the displacement-load curves are nonlinear, and the displacement increases gradually with the increase of load on the posterior AF. From Figure 2e, it can be seen that inner AF exhibits lower radial displacement than outer AF. From Figure 2f, it can be seen that upper AF exhibits lower radial displacement than lower AF. The radial displacement state is in good agreement with the radial displacement field quite well, as shown in Figure $2 b$.

\section{Discussion}

The displacement state of the posterior annulus in the intervertebral disc was analysed noninvasively using the optimized DIC technique, and it is found that there is a significant bulging phenomenon in the posterior annulus of the intervertebral disc under flexion.

The first finding is that the average axial displacement is compressive in posterior $\mathrm{AF}$, and the axial displacement in the outer region is significantly lower than that in the inner region, while the axial displacement in the lower region is significantly lower than that in the upper region. This result is in good agreement with early research on the strain distribution in the intervertebral disc under compression load [24,25]. In addition, it should be noted that the difference in the displacement in different regions is even more significant under flexion than under compression. These findings may have physiological importance. As can be seen from Equation 5, the increase of axial pressure on the intervertebral disc under flexion leads to a significant reduction in the height as well as the across sectional area in the inner region of the posterior AF, resulting in the increase of NP pressurization. These factors may contribute to the initiation or propagation of radial tears in the inner region of the posterior $\mathrm{AF}$, leading to lumber disc herniation.

The second finding is that the average radial displacement is tensile in posterior $\mathrm{AF}$, and the radial displacement in the inner region is significantly lower than that in the outer region, while the radial displacement in the upper region is significantly lower than that in the lower region. As can be seen from Equation 6, the increase of radial shear force leads to the shift of the intervertebral disc in the horizontal plane, resulting in an exacerbation of lumbar disc herniation. In addition, the outward bulge in the outer AF may be caused by the shift of the nucleus pulposus under load condition. It is thought that radial tearing begins at the inner $\mathrm{AF}$ and progresses toward the external boundaries and the huge stretching in the outer AF may lead to the radial tears and the herniation [26,27].

\section{Conclusions}

The optimized digital image correlation technique was applied to measure the internal displacement of the intervertebral disc under axial compression with flexion position. Firstly, the results show that the average axial displacement is compressive in posterior AF, and the axial displacement in the outer region is significantly lower than that in the inner region, while the axial displacement in the lower region is significantly lower than that in the upper region. Secondly, it is found that the average radial displacement is tensile in posterior AF, and the radial displacement in the inner region is significantly lower than that in the outer region, while the radial displacement in the upper region is significantly lower than that in the lower region.

\section{Acknowledgements}

The project is partly supported by the National Natural Science Foundation of China (No. 11672208, 11432016 and 81741141) and the Natural Science Foundation of Tianjin City (No. 17JCQNJC14200).

\section{References}

1. Provaggi E, Leong JJ, Kalaskar DM. Applications of 3D printing in the management of severe spinal conditions. Proc Inst Mech EngJ Eng Med 2016; 231.

2. Abdu RW, Abdu WA, Pearson AM. Reoperation for recurrent intervertebral disc herniation in the spine patient outcomes research trial: analysis of rate, risk factors and outcome. Spine 2017; 42: 1106-1114.

3. Lurie JD, Henderson ER, Mcdonough CM. The effect of expectations on treatment outcome for lumbar intervertebral disc herniation. Spine 2016; 41: 803.

4. Leven DM, Passias PG, Errico TJ. Risk factors for reoperation in patients treated surgically for intervertebral disc herniations: a subanalysis of the eight-year data from the SPORT trial. Spine J 2016; 41: 901.

5. Bai J, Zhang W, Wang Y. Application of transiliac approach to intervertebral endoscopic discectomy in L5/S1 intervertebral disc herniation. Eur J Med Res 2017; 22: 22.

6. Tsarouhas A, Soufla G, Tsarouhas K. Molecular profile of major growth factors in lumbar intervertebral disc herniation: correlation with patient clinical and 
epidemiological characteristics. Mol Med Rep 2017; 15: 2195-2203.

7. Casaroli G, Villa T, Bassani T. Numerical prediction of the mechanical failure of the intervertebral disc under complex loading conditions. Materials 2017; 10: 31 .

8. Laws CJ, Bergjohansen B, Hargens AR. The effect of simulated microgravity on lumbar spine biomechanics: an in vitro study. Eur Spine J 2016; 1-9.

9. Nagel TM, Zitnay JL, Barocas VH. Quantification of continuous in vivo flexion-extension kinematics and intervertebral strains. Eur Spine J 2014; 23: 754-761.

10. Schechtman H, Robertson PA, Broom ND. Quasi-static and cyclic compressive loading studies of the intervertebral disc with combined flexion and torsion. Revista Brasileira De Engenharia Biomedica 2012; 28: 311-318.

11. Yu B, Zhang C, Qin C. FE modeling and analysis of L4-L5 lumbar segment under physiological loadings. Technol Health Care 2015; 23: 383-396.

12. Bergjohansen B, Fields AJ, Liebenberg EC. Cartilage endplate thickness variation measured by ultrashort echotime MRI is associated with adjacent disc degeneration. J Orthop Res 2017; 9999: 1-10.

13. Peloquin JM, Santare MH, Elliott DM. Advances in quantification of meniscus tensile mechanics including nonlinearity, yield, and failure. J Biomech Eng 2016; 138: 021002.

14. Yoder JH, Peloquin JM, Song G. Internal three-dimensional strains in human intervertebral discs under axial compression quantified noninvasively by magnetic resonance imaging and image registration. J Biomech Eng 2014; 136: 111008 .

15. Palanca M, Brugo TM, Cristofolini L. Use of digital image correlation to investigate the biomechanics of the vertebra. J Mech Med Biol 2015; 15: 1540004.

16. Ryan G, Pandit A, Apatsidis D. Stress distribution in the intervertebral disc correlates with strength distribution in subdiscal trabecular bone in the porcine lumbar spine. Clin Biomech 2008; 23: 859-869.

17. Little JP, Pearcy MJ, Tevelen G. The mechanical response of the ovine lumbar anulus fibrosus to uniaxial, biaxial and shear loads. J Mech Behav Biomed Mater 2010; 3: 146-157.

18. Little JP, Pearcy MJ, Izatt MT. Understanding how axial loads on the spine influence segmental biomechanics for idiopathic scoliosis patients: a magnetic resonance imaging study. Clin Biomech 2015; 32: 220-228.
19. Hollingsworth NT, Wagner DR. The stress and strain states of the posterior annulus under flexion. Spine 2012; 37: 1134.

20. Shan Z, Wade KR, Schollum ML. A more realistic disc herniation model incorporating compression, flexion and facet-constrained shear: a mechanical and microstructural analysis. part II: high rate or surprise loading. Eur Spine J 2017; 1-13.

21. OConnell GD, Vresilovic EJ, Elliott DM. Human intervertebral disc internal strain in compression: the effect of disc region, loading position, and degeneration. J Orthop Res 2015; 29: 547-555.

22. Ryan G, Pandit A, Apatsidis D. Stress distribution in the intervertebral disc correlates with strength distribution in subdiscal trabecular bone in the porcine lumbar spine. Clin Biomech 2008; 23: 859 .

23. Gao LL, Zhang CQ, Gao H. Depth and rate dependent mechanical behaviors for articular cartilage: experiments and theoretical predictions. Mater Sci Eng 2014; 38: 244-251.

24. Qing L, Tai-Yong W, Xiu-Ping Y. Strain distribution in the intervertebral disc under unconfined compression and tension load by the optimized digital image correlation technique. Proc Inst Mech Eng 2014; 228: 486-493.

25. OConnell GD, Vresilovic EJ, Elliott DM. Human intervertebral disc internal strain in compression: the effect of disc region, loading position, and degeneration. J Orthop Res 2011; 29: 547-555.

26. Haefeli M, Kalberer F, Saegesser D. The course of macroscopic degeneration in the human lumbar intervertebral disc. Spine 2006; 31: 1522-1531.

27. Vernonroberts B, Fazzalari NL, Manthey BA. Pathogenesis of tears of the anulus investigated by multiple-level transaxial analysis of the T12-L1 disc. Spine 1997; 22: 2641-2646.

\section{*Correspondence to}

Chun-Qiu Zhang

Tianjin Key Laboratory for Advanced Mechatronic System Design and Intelligent Control

School of Mechanical Engineering

Tianjin University of Technology

PR China 